\title{
PARTICIPATION OF CHELATING METALS IN CARBOXYLATION REACTIONS
}

\author{
Martin Stiles \\ Department of Chemistry, University of Michigan, Ann Arbor, Mich.
}

\section{Introduction}

Chelate compounds, arising out of the combination of inorganic ions with polyfunctional organic molecules, may be expected to hold great interest for investigators in widely different fields of chemistry. The stability of many such substances can be an important factor both in the manipulation of ionic equilibria and in the alteration of organic structures. In the former application there has been great progress in recent years, so that the use of polyfunctional organic compounds as precipitants, titrants, and solubilizing agents for metals is now common. Alteration of the effective emf of a redox system such as represented in EQUATION 1, by addition of a chelating agent that will preferentially bind one of the metal ions (EQUATION 2), is likewise familiar.

$$
\begin{aligned}
M^{n}+[\mathrm{Ox}] & \rightleftharpoons M^{n+1}+[\mathrm{Red}] \\
M^{n+1}+\mathrm{A}-\mathrm{B} & \rightleftharpoons\left[\begin{array}{c}
A-B \\
/ \\
M
\end{array}\right]^{n+1} \\
A+B & \rightleftharpoons A-B
\end{aligned}
$$

Use of analogous techniques to influence organic reactions has lagged far behind. ${ }^{1}$ The importance of chelating metals in many of the organic reactions that occur in biological systems, though recognized, is poorly understood. Westheimer's clarification ${ }^{2}$ of the catalytic effect of chelating metal ions upon the decarboxylation of oxaloacetic acid did not have synthetic utility, but it pointed to the potential importance of chelating metals in organic reactions in general. A very direct kind of influence which a chelating metal could have upon a reversible organic reaction is illustrated by EQUATIONS 2 and 3, where the formation of a stable chelate (EQUATION 2) serves to displace the equilibrium of EQUATION 3 in favor of the bifunctional product $A-B$. The improved yields in the preparation of certain chelating agents that result when copper ions are added to the reaction mixture ${ }^{1}$ and the similar effect that borate recently has been reported ${ }^{3}$ to have upon the formation of $p$-aminosalicylic acid may be examples of this "equilibrium effect" of chelation.

It was desirable to test the synthetic utility of this phenomenon in a more direct fashion by investigating a reversible reaction that otherwise fails, that is, one whose equilibrium position lies completely on the side of monofunctional compounds ( $A$ and $B$ in EqUation 3 ). The reaction chosen ${ }^{4}$ was that of EQUATION 4. Steinkopf had shown ${ }^{5}$ that nitroacetic acid decarboxylated completely,

$$
\mathrm{O}_{2} \mathrm{~N}-\mathrm{CH}_{2}-\mathrm{CO}_{2} \mathrm{H} \underset{?}{\stackrel{\leftrightarrow}{\rightleftarrows}} \mathrm{O}_{2} \mathrm{~N}-\mathrm{CH}_{3}+\mathrm{CO}_{2}
$$


and no evidence had appeared since then to suggest that the reaction ever proceeded in the opposite direction.

The evidence that nitroacetic acid (I) formed chelate salts was limited to Pedersen's observation ${ }^{6}$ that certain metal ions (for example, $\mathrm{Al}^{+3}, \mathrm{Cu}^{+2}, \mathrm{Mg}^{+2}$ ) retarded appreciably the rate of the decarboxylation reaction. It was found ${ }^{4,7}$ that the aluminum and magnesium salts of $I$ could be prepared in methanol or ether, and comparison of their spectra and stability characteristics with those of the alkali metal salts led to the proposal of the chelate structure (II). The magnesium salt (II, $M=\mathrm{Mg}$ ) can be easily prepared by mixing equivalent

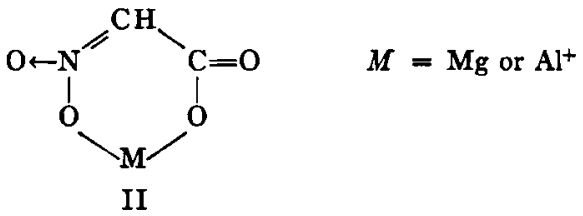

quantities of nitroacetic acid (I) and magnesium methoxide in methanol. The resulting solution is stable for hours at room temperature, in sharp contrast to solutions prepared in identical fashion from I and sodium methoxide. ${ }^{7.8}$ Even the magnesium salt is quite sensitive to acid, however, being partially decarboxylated by even so weak an acid as carbon dioxide in methanol.?

\section{Carboxylation with Magnesium Methyl Carbonale}

Consideration of the properties of the chelate salts (II) made it possible to design experiments aimed at using chelation to reverse the reaction of EQUATION 4. This objective was accomplished in principle when the spectrum of II was observed to develop during experiments in which nitromethane was treated with magnesium methoxide (or a mixture of aluminum and sodium alkoxides) and carbon dioxide in methanol. ${ }^{7}$ Careful hydrolysis of such solutions led to isolable quantities of nitroacetic acid (I). Further modification of the technique led to the development of magnesium methyl carbonate in dimethylformamide as a reagent for the carboxylation of primary nitroparaffins in yields that are satisfactory from a preparative point of view. Nitroparaffins that have been successfully carboxylated with this reagent include nitromethane, ${ }^{4}$ nitroethane, ${ }^{4} 1$ - nitropropane, ${ }^{4} 1$ - nitrobutane, ${ }^{4} 1$ - nitro-3-methylbutane (Stiles, unpublished experiments), and 3- $\beta$-nitroethylindole. ${ }^{8}$

Subsequently it was shown ${ }^{9}$ that magnesium methyl carbonate (MMC) could be used to carboxylate ketones which contain enolizable methyl or methylene groups (EQUATION 5). The intermediate chelate salts of $\beta$-keto acids (III)

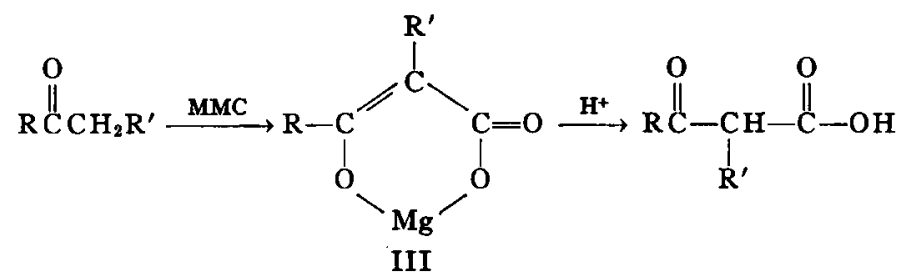

were characterized by their intense absorption in the ultraviolet and by the 
fact that they could be alkylated and acylated at the $\alpha$-carbon (see below). Ketones that have been carboxylated by this technique include acetophenone, ${ }^{9}$ 1-tetralone, ${ }^{9}$ 1-indanone, ${ }^{9}$ cyclohexanone, ${ }^{9}$ and cyclopentanone.$^{8}$

\section{Role of Magnesium Ions in the Carboxylation Reactions}

It is important to recognize that the success of the carboxylation technique just described rests on a kinetic effect exerted by the magnesium ions in addition to the simple equilibrium effect referred to earlier. The equilibrium of EQUATION 6 is completely to the left in all solvents studied ${ }^{6,7}$ - water, methanol, and ether. One has clearly, therefore, to overcome this unfavorable equilibrium

$$
\begin{array}{ll}
\mathrm{CH}_{2} & =\mathrm{NO}_{2}^{-}+\mathrm{CO}_{2} \rightleftharpoons \mathrm{O}_{2} \mathrm{NCH}_{2} \mathrm{CO}_{2}^{-} \\
\stackrel{\mathrm{O}}{\mathrm{O}^{-}} & \\
\mathrm{R}-\mathrm{C}=\mathrm{CH}_{2} & +\mathrm{CO}_{2} \rightleftharpoons \mathrm{R}-\stackrel{\mathrm{C}}{-}-\mathrm{CH}_{2} \mathrm{CO}_{2}{ }^{-}
\end{array}
$$

in the carboxylation reaction by conversion of the unstable monoanion to the chelate salt (II). This function of the metal ion may be designated the equilibrium effect. This same factor may be assumed to facilitate conversion of the enolate of a ketone to the $\beta$-keto acid salt (EQUATION 7) since there is good evidence that chelate salts (III) intervene in this system also. However, it must be recognized that the equilibrium of EQUaTION 7 lies much further to the right, in an inert solvent, than that of EQUATION $6,{ }^{*}$ so much so that $\beta$ keto acids can be prepared in fair yields by treating an ether solution of the sodium or potassium enolate with carbon dioxide. ${ }^{11,12}$ The equilibrium effect of magnesium ions is therefore not sufficient to explain the effectiveness of magnesium methyl carbonate in carboxylating ketones. The explanation of the additional kinetic effect appears to lie in the greater tendency of magnesium ions, compared to the alkali metals, to coordinate with the carboxyl groups of the reagent. The result is to make the carbonyl group of magnesium methyl carbonate (IV) resemble that of a carbonate ester (V) and hence susceptible to nucleophilic attack, in contrast to the inert carboxylate ion (VI). $\dagger$ Thus<smiles>CCOC(=O)O[Mg]</smiles>

IV<smiles>COC(=O)OC</smiles>
$\mathrm{V}$<smiles>COC(=O)[CH-]C(=O)[O-]</smiles>

VI

* In aqueous solution, bicarbonate ion and ketone or nitroparaffin are the stable species, so that decarboxylation is favored thermodynamically in both systems. However, in an aprotic solvent such as ether (and presumably in dimethylformamide) the relative positions of equilibrium are as stated above. It should be pointed out that the rates of decarboxylation in the two systems depend on acidity in opposite ways; the anion of an $\alpha$-nitro acid is the reactive species, ${ }^{6}$ while the free $\beta$-keto acid decarboxylates more rapidly than its alkali metal salt.10 Nevertheless, both decarboxylations ultimately go to completion in aqueous solution regardless of whether the medium is acidic or alkaline.

$\dagger$ An alternative explanation of the kinetic effect is based upon the possibility that free carbon dioxide, present in the reagent, is the species which reacts with the enolate ion to 
the presence of magnesium ions gives rise to a carboxylating species (IV) that is compatible with the basic catalyst (low concentrations of methoxide) necessary to generate the enolate ion from the ketone. Such a system can therefore be used as a one-step carboxylating reagent, and for this reason it is usually more satisfactory than the two-step method required in the absence of a chelating metal, particularly since the very strongest bases (sodium amide or triphenylmethide) must be used in the older technique.

\section{Alkylation of Chelate Salts}

The enolate structure assigned to the chelate salts (II and III) suggests that the substances should be reactive toward alkylating and acylating agents. This was found ${ }^{9}$ to be the case for the $\beta$-keto acid derivatives (III). Addition of a reactive alkyl halide to the reaction product from a ketone and magnesium methyl carbonate leads to alkylation of the $\alpha$-carbon atom. In some instances at least (EQUATION 8) the method is a practical synthetic procedure. ${ }^{9}$ The success of this synthetic scheme tends to confirm the structure assigned to the
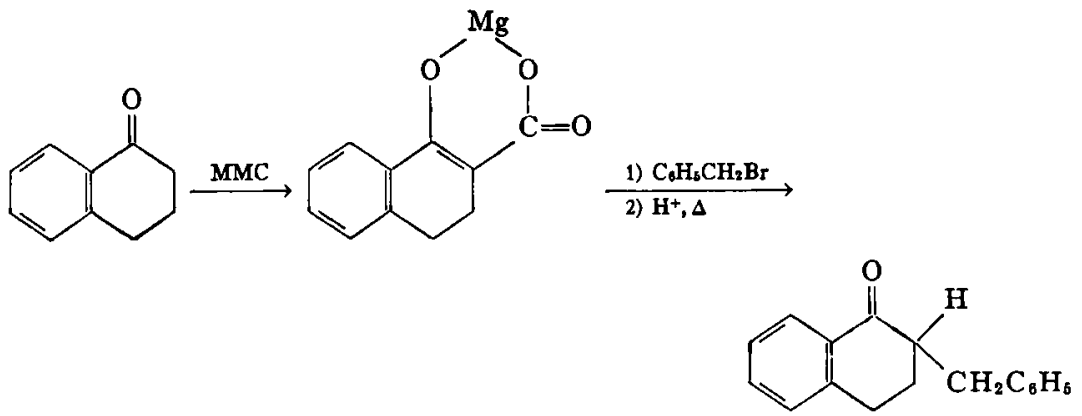

chelate salts. At the same time it emphasizes the way in which the magnesium salt of a $\beta$-keto acid can resemble a $\beta$-keto ester, a similarity discussed in the preceding section. Treatment of the chelate salts (III) with an acylating agent such as benzoyl chloride leads in similar fashion to attack at the $\alpha$-carbon and ultimately to $\beta$-diketones (Stiles, unpublished experiments). However, the acylation reaction is complicated by the reaction between acid chlorides and the MMC reagent, so that it is not at present a useful synthetic procedure.

Treatment of the $\alpha$-nitro acid salts (II) with ordinary alkyl halides has not resulted in the desired alkylation reaction. Attack of the halide in this case appears to occur at one of the oxygen atoms of the nitro group, leading to oxidation of the halide. Similar results have been reported in the attempted alkylation of nitroparaffin salts. ${ }^{13}$ Use of gramine methiodide as the alkylating agent for the salt II has been successful, presumably because of the different mechanism by which this alkylating agent functions. ${ }^{14}$ Thus nitromethane could be converted to the chelate (II), alkylated with gramine methiodide, and decarboxylated to furnish a high yield of $3-\beta$-nitroethylindole. ${ }^{7,8}$

produce $\beta$-keto acid salt. Magnesium methyl carbonate solutions are known to evolve $\mathrm{CO}_{2}$ at much lower temperatures than sodium methyl carbonate, which is ineffective toward ketones. Current work is directed toward deciding this point. 


\section{Enzymatic Carboxylation Reactions}

It is of interest to extend some aspects of the foregoing discussion to enzymatic carboxylation reactions. The reactions of direct interest will be those in which an "active hydrogen compound" such as a ketone or thiol ester, or a vinylog of such compound, is carboxylated at one of the enolizable positions. It is reasonable to assume that the actual bond-making process resembles, electronically, that of the nonenzymatic reaction, that is, it is a nucleophilic attack by an enol or enolate species upon the carbon atom of carbon dioxide or one of its derivatives, similar to the process of EQUATION 9. This representa-<smiles>[R]C(=O)C(C(=O)[O-])C(=O)[O-]</smiles>

tion must be modified, however, to take into account the interaction of the reactants with the enzyme.

Although few precise data are available on the variation of reaction rates with carbon dioxide concentration in enzymatic reactions, there are at least qualitative indications ${ }^{15-17}$ that the Michaelis-Menten law $^{18}$ is obeyed, and that $\mathrm{CO}_{2}$ must be bound to the enzyme prior to its attachment to the substrate.* If one centers attention on the carbon dioxide reactant, it is therefore necessary to account for two events: (1) the binding of $\mathrm{CO}_{2}$ (or a suitable derivative) to the enzyme, and (2) the transfer of the bound $\mathrm{CO}_{2}$ to the substrate, which is assumed to be suitably activated and oriented by the enzyme. These two events can be unrelated chemically when the species under consideration is a large polyfunctional molecule, as is so frequently the case, but the small size and essentially monofunctional nature of carbon dioxide require that the two processes be closely connected. This connection can provide an insight into the nature of the binding, assuming only that the chemical behavior of $\mathrm{CO}_{2}$ closely parallels that observed in nonenzymatic reactions.

The simplest mode of attachment of carbon dioxide would involve a single site that, in principle, could be either acidic (EQUATION 10) or basic (EQUATION

$$
\begin{array}{ll}
\mathrm{CO}_{2}+{ }^{(+)} \mathrm{A}-\xi \rightarrow \mathrm{O}=\mathrm{C}=\stackrel{(+)}{\mathrm{O}}-\mathrm{A}-\xi \\
\mathrm{CO}+{ }^{(-)} \mathrm{B}-\xi \rightarrow{ }_{(-)}^{\mathrm{O}} \rightarrow \mathrm{C}-\mathrm{B}-\xi
\end{array}
$$

11). The former binding is very unlikely; nowhere in the chemistry of carbon

* The possibility that $\mathrm{CO}_{2}$ might be incorporated into a coenzyme prior to interaction with the apoenzyme does not alter the argument that follows; it would then be the $\mathrm{CO}_{2}$-coenzyme binding that concerned us. In those cases ${ }^{16,10}$ where the saturation level of carbon dioxide is unusually high, one might suspect that an unfavorable equilibrium between carbon dioxide and some other substance present precedes the enzyme-catalyzed step. For example:

$$
\begin{aligned}
\mathrm{CO}_{2}+\mathrm{X} & \rightleftharpoons \mathrm{XCO}_{2} \\
\mathrm{XCO}_{2}+\text { Enzyme } & \rightleftharpoons \text { Complex }
\end{aligned}
$$


dioxide can one find evidence that the substance functions as a base. ${ }^{*}$ The second possibility (EQUATION 11) is inherently very likely, for carbon dioxide combines energetically with bases of many varieties, but the resulting adduct cannot be expected to exhibit the required reactivity toward an enol or similar nucleophilic substrate. As pointed out in one of the earlier paragraphs, carboxylate ions show little tendency to enter into this type of addition reaction. The coordination of a proton or metal ion with the carboxylate ion to give a species such as VII (or the formation of an equivalent structure by combina-<smiles>O=C([Hg])O[Na]</smiles>

VII

tion of bicarbonate with the enzyme) would lend the desired reactivity, but the acidity of VII (where B is oxygen or nitrogen) would be greater than that of acetate, for example, and hence the dissociation to the inert anion would be virtually complete in water at $p \mathrm{H} 7$.

The foregoing argument leads to the conclusion that carbon dioxide needs to be attached to the enzyme at two sites (EQUATION 12) in order to be both bound and reactive.

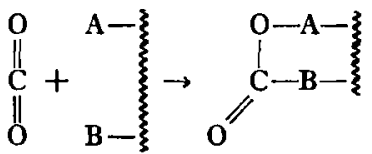

VIII

The only experimental evidence to date on the nature of a $\mathrm{CO}_{2}$-enzyme complex is that recently furnished by Lynen and his collaborators. ${ }^{22}$ These investigators have shown that $\beta$-methylcrotonyl CoA carboxylase contains biotin units that are utilized in the catalytic action of the enzyme and, further, that the enzyme can be induced to catalyze the introduction of a carboxyl group onto one of the nitrogen atoms of free biotin. They have reasonably concluded that the biotin- $\mathrm{CO}_{2}$ compound that they characterized is representative of the active carboxylating agent. Consideration of the detailed structure of biotin- $\mathrm{CO}_{2}$ (partial structure $\mathrm{IX}$ ), including the indubious chelate ring, shows<smiles></smiles>

$\mathrm{IX}$

* The solubility of carbon dioxide in sulfuric acid is approximately the same as in water; less, in fact, than in various hydrocarbon solvents. ${ }^{20,21}$ Experiments designed to discover specific interactions between $\mathrm{CO}_{2}$ and $\mathrm{BF}_{\mathrm{g}}$ in the gas phase gave no evidence for such interaction (R. S. Berry, private communication). 
that it fulfills the requirements outlined above for reactive carbon dioxide. Although no requirement for a chelating metal is associated with the function of Lynen's enzyme-biotin- $\mathrm{CO}_{2}$ complex, ${ }^{22}$ the general need for such metals in enzymatic carboxylations of varied type ${ }^{23}$ suggests that instances may be found in which a metal ion fulfills the role played by the chelated hydrogen in IX (that is, A in formula VIII).

Recent work on the enzymatic synthesis of fatty acids ${ }^{15,24,25}$ has revealed a pattern of steps in which carboxylation of an "active hydrogen" compound is followed by such reactions as acylation, decarboxylation, and reduction, as summarized in EQUATION 13. The similarity of this sequence to the pattern<smiles>[X]C(=O)CC(=O)SCC(=O)CC(=O)ON(C(=O)O)C(=O)O</smiles><smiles>CCCOC(=O)CC(=O)CCC</smiles>

XII

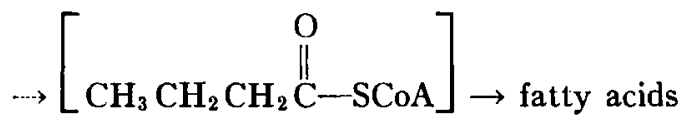

of acylation and alkylation reactions that has been observed to involve chelate salts (EQUATION 8, for example) is striking. The involvement of malonyl CoA (XI) in chelation would provide an attractive rationale for its intervention in this biosynthetic scheme. In fact, a very recent laboratory synthesis for substituted $\beta$-keto esters furnishes a precise model for this series of transformations. Ireland and Marshall ${ }^{26}$ have found that monoesters of malonic acids (XIII) can be converted to chelate salts XIV, which are readily acylated and decarboxylated to give the $\beta$-keto ester product $\mathrm{XV}$.

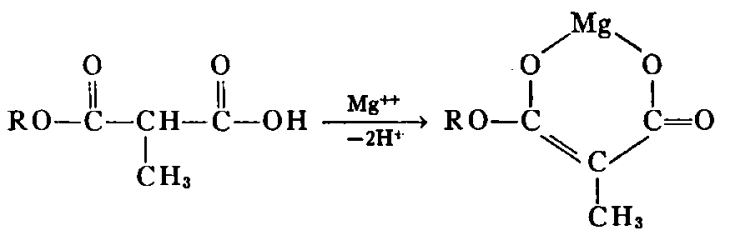




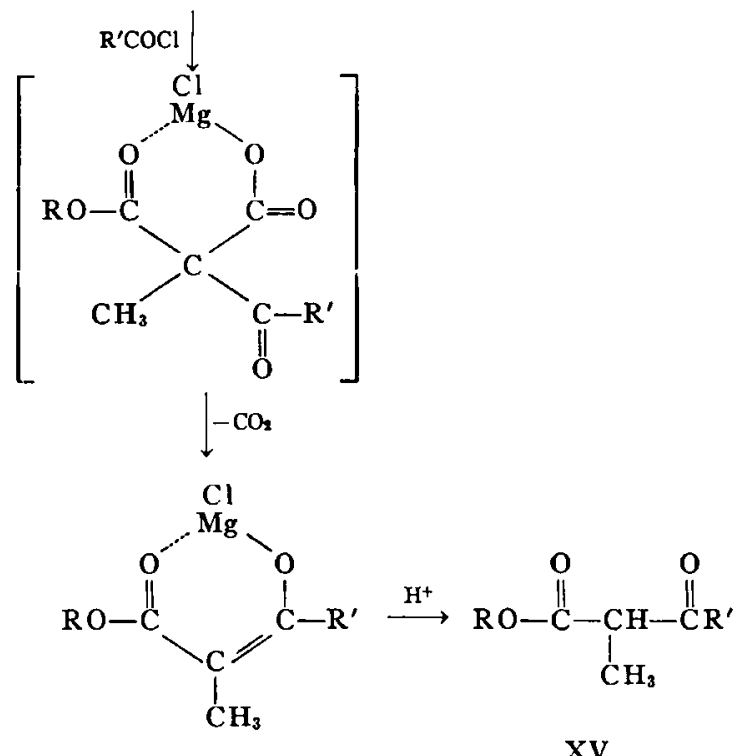

It is obvious that the occurrence of chelation phenomena in organic reactions goes far beyond the examples that have been discussed here. One can expect to find that in the future, chelation will find increasing use in the deliberate control of organic transformations. A thorough understanding of the importance of chelation in biological systems, even in the limited field of carboxylation reactions, must await more precise information from studies of the chemical effects of chelation, as well as from studies of intermediate structures in enzyme reactions.

\section{References}

1. Marteil, A. E. \& M. Calvin. 1952. Chemistry of the Metal Chelate Compounds. : 421-424. Prentice-Hall. New York, N. Y.

2. Steinberger, R. \& F. H. Westheimer. 1949. J. Am. Chem. Soc. 71: 4158 . Also 1951. 73: 429 .

3. Doub, L., J. A. Schaefer, O. L. Stevenson, C. T. Walker \& J. M. Vandenbelt. 1958. J. Org. Chem. 23: 1422.

4. Stiles, M. \& H. L. Finkbeiner. 1959. J. Am. Chem. Soc. 81: 505.

5. StEINKOPF, W. 1909. Berichte deutschen chem. Gesell. 42: 2026, 3925.

6. Pedersen, K. J. 1927. Trans. Faraday Soc. 23: 316. Also 1949. Acta Chem. Scand. 3: 676.

7. FinKBeiner, H. L. 1959. Thesis. Univ. Mich. Ann Arbor, Mich.

8. Stiles, M. \& H. L. Finkbeiner. 1959. Symposium on Nitroparaffins, Am. Chem. Soc. Meeting, September 15, Atlantic City, N. J.

9. Stiles, M. 1959. J. Am. Chem. Soc. 81: 2598.

10. WidMark, E. M. P. 1920 . Acta Med. Scand. 63: 393.

11. Baumgarten, E., R. Levine \& C. R. Hauser. 1944. J. Am. Chem. Soc. 66: 862.

12. Levine, R. \& C.'R. Hauser. 1944. J. Am. Chem. Soc. 66: 1768.

13. HASS, H. B. \& M. L. Bender. 1949. J. Am. Chem. Soc. 71: 1767, cite several examples.

14. Albright, J. D. \& H. R. SNyder. 1959. J. Am. Chem. Soc. 81: 2239.

15. Gibson, D. M., E. B. Titchener \& S. J. Wakil. 1958. J. Am. Chem. Soc. 80: 2908.

16. Bachhawat, B. K. \& M. J. Coon. 1958. J. Biol. Chem. 231: 625.

17. TChen, T. T. \& B. Vennesland. 1955. J. Biol. Chem. 213: 533. 
18. LaIDler, K. J. 1954. Introduction to the Chemistry of Enzymes. : 18-19. McGrawHill, New York, N. Y.

19. Flavin, M., H. Castro-Mendeza \& S. OchoA. 1956. Biochim. et Biophys. Acta. 20: 591 .

20. Christoff, A. 1906. Z. physik. Chem. 55: 627.

21. Just, G. 1901. Z physik. Chem. 37: 342.

22. Lynen, F., J. Knappe, E. Lorch, G. Jütring \& E. Ringlemann. 1959. Angew. Chem. 71: 481-486.

23. Froton, J. S. \& S. Simmonds. 1959. General Biochemistry. Wiley. New York, N. Y.

24. Wakil, S. J. 1958. J. Am. Chem. Soc. 80: 6465.

25. Formica, J. V. \& R. O. Brady. 1959. J. Am. Chem. Soc. 81: 752.

26. Ireland, R. E. \& J. A. Marshall. 1959. J. Am. Chem. Soc. 69: 2907. 